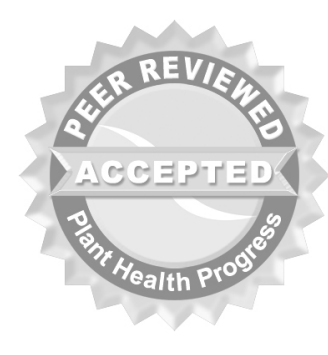

(c) 2009 Plant Management Network.

Accepted for publication 23 April 2009. Published 12 J une 2009.

\title{
A New Canker Disease of Crabapple Trees Caused by Phacidiopycnis washingtonensis in Washington State
}

\author{
C. L. Xiao, Y. K. Kim, and R. J . Boal, Tree Fruit Research and \\ Extension Center, Washington State University, Wenatchee, WA \\ 98801
}

Corresponding author: C. L. Xiao. clxiao@wsu.edu

Xiao, C. L., Kim, Y. K., and Boal, R. J. 2009. A new canker disease of crabapple trees caused by Phacidiopycnis washingtonensis in Washington state. Online. Plant Health Progress doi: 10.1094/PHP-2009-0612-01-BR.

Crabapple (Malus sylvestris Mill.) is commonly used as a source of pollen in apple production. In September 2003, a canker and twig dieback disease of 'Manchurian' crabapple trees was observed in a commercial 'Gala' apple (Malus domestica Borkh.) orchard in Manson, WA. An unidentified fungus was consistently isolated from $20 \%$ of sampled crabapple twigs exhibiting cankers or dieback symptoms, while Sphaeropsis pyriputrescens was isolated from the remaining diseased twigs. The same symptoms and fungal association also were observed on crabapple twigs in a 'Red Delicious' apple orchard in Royal City, WA, during May 2004 and in a 'Fuji' apple orchard in Manson, WA, during May 2004 and 2005. In March 2008, the same symptoms again were observed on the vast majority of the crabapple trees in two Fuji orchards in Brewster, WA, and the same fungus was isolated from infected twigs of four randomly sampled trees. Canker and dieback appeared to originate from infections of fruit spurs or pruning wounds (Fig. 1). Cankered areas were slightly sunken, brown to dark. Pycnidia were often present in aged areas of the cankers. Pycnidia were black, 0.3 to $0.5 \mathrm{~mm}$ in diameter, and partially immersed in the diseased tissue (Fig. 2). To isolate the fungus, outer bark tissues of diseased twigs were scraped and small tissue segments were cut from the canker margin. Tissue segments were surface-disinfested for $5 \mathrm{~min}$ in $0.5 \%$ sodium hypochlorite solution, rinsed three times with sterile water, cut into small pieces and placed on acidified potato-dextrose agar (APDA, $4.0 \mathrm{ml}$ of a $25 \%$ solution of lactic acid per liter of medium). Isolation plates were incubated at $20^{\circ} \mathrm{C}$ in the dark. The colonies of the fungus first appeared white and then light gray with fluffy aerial mycelium, and alternating rings of dark gray and light gray were evident in cultures particularly on the reverse of petri plates. Cream-colored conidial masses at pycnidial ostioles were commonly present in 1- to 2-week-old cultures grown at $20^{\circ} \mathrm{C}$ in the dark. Conidia were hyaline, lacriform with the dehiscence end flattened or ovoid to ellipsoid without the cicatrice, 6-7.5 ×3-4 $\mu$ m (Fig. 3). The fungus was identified as Phacidiopycnis washingtonensis Xiao \& J. D. Rogers (3). To complete Koch's postulates, two isolates (one from diseased crabapple twig and one from decayed apple fruit) were used in pathogenicity tests on Manchurian crabapple trees. In the orchard, selected 2-year-old twigs were sprayed with $70 \%$ ethanol and allowed to dry. Twigs were wounded to a depth of 1 to $2 \mathrm{~mm}$ with a sterile 5-mm-diameter cork borer, and a 5-mm mycelial plug from a 6-day-old PDA culture of P. washingtonensis was placed into each wound. Twigs wounded and treated with sterile PDA plugs were used as controls. Inoculation sites were covered with moist cheesecloth and sealed with Parafilm, which was removed 3 weeks after inoculation. Four twigs per isolate on each of four trees were inoculated. The inoculation was conducted in February 2006 in two orchards at different locations. At 2 months after inoculation all twigs were removed, canker sizes were measured, and reisolation of the fungus was attempted as described above. Both isolates caused cankers on crabapple twigs. Canker sizes at 2 months after inoculation ranged 
from 6.3 to $16.5 \mathrm{~mm}$ with means of 9.2 and $7.1 \mathrm{~mm}$ for location 1 and location 2, respectively. No cankers developed on control twigs. P. washingtonensis was reisolated from all except one inoculated twig and was not recovered from noninoculated controls. P. washingtonensis is the cause of a recently recognized postharvest fruit rot disease of apple (1,3). This is the first report of this fungus causing cankers and twig dieback on crabapple trees. Our findings indicate that canker symptoms on crabapple trees caused by P. washingtonensis are similar to those caused by S. pyriputrescens (2) and that infected crabapple trees may serve as a source of P. washingtonensis inoculum for infection of apple.

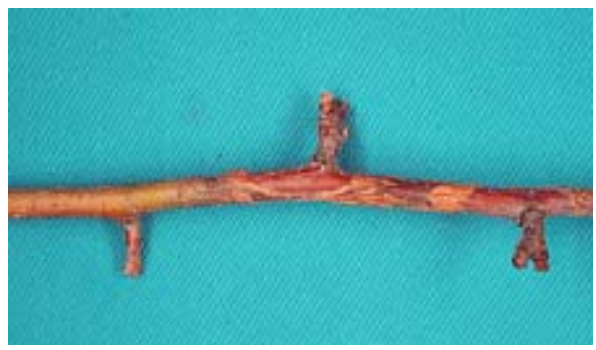

Fig. 1. Canker symptoms on crabapple twigs caused by Phacidiopycnis washingtonensis.

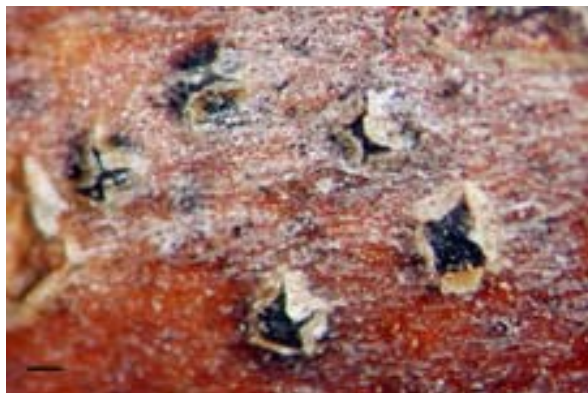

Fig. 2. Close-up of pycnidia of Phacidiopycnis washingtonensis on a diseased twig of crabapple. Scale bar $=0.25 \mathrm{~mm}$.

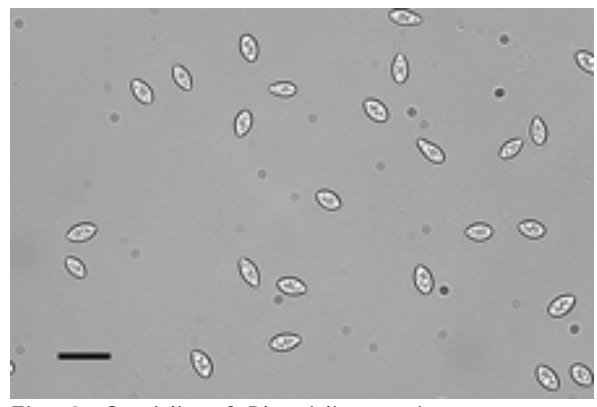

Fig. 3. Conidia of Phacidiopycnis washingtonensis. Scale bar $=10 \mu \mathrm{m}$.

\section{Literature Cited}

1. Kim, Y. K., and Xiao, C. L. 2006. A postharvest fruit rot in apple caused by Phacidiopycnis washingtonensis. Plant Dis. 90:1376-1381.

2. Xiao, C. L., and Boal, R. J. 2005. A new canker and twig dieback disease of apple and crabapple trees caused by Sphaeropsis pyriputrescens in Washington State. Plant Dis. 89:1130.

3. Xiao, C. L., Rogers, J. D., Kim, Y. K., and Liu, Q. 2005. Phacidiopycnis washingtonensis: A new species associated with pome fruits from Washington State. Mycologia 97:464-473. 